\title{
Carotenoids, Polyphenols and Antioxidant Activity Evaluation in Stone-Grinded Wheat Semolina
}

\author{
Pamela Vignolini ${ }^{1}$, Silvia Urciuoli ${ }^{1}$, Daniela Heimler ${ }^{2}$ and Annalisa Romani ${ }^{1}$ \\ 1. Laboratorio Phytolab, DiSIA, Dipartimento di Statistica, Informatica, Applicazioni “Giuseppe Parenti”, Università degli Studi di \\ Firenze,Polo Scientifico e Tecnologico via U. Schiff, 6-Sesto Fiorentino (FI) 50019, Italy \\ 2. PIN S.c.r.l.-Servizi Didattici e Scientifici per l'Università di Firenze, Piazza G. Ciardi, 25, Prato 59100, Italy
}

Summary: The functional quality of stone grinded grain semolina, also related to the presence of secondary antioxidant metabolites, was evaluated by HPLC/DAD (high performance liquid chromatography/diode array detection) analysis and by in vitro spectrophotometric techniques. The total polyphenol content was determined with the Folin-Ciocalteau spectrophotometric method, while carotenoid content evaluation was performed by HPLC/DAD chromatographic analysis: the presence of lutein as the main compound ranging between 75 and $78.5 \%$ of total carotenoids was pointed out. The chromatographic analyses identified the presence of an indolacetic acid derivative whose content changed between 0.097 and $0.108 \mathrm{mg} / \mathrm{g}$.

Key words: HPLC/DAD, lutein, Folin-Ciocalteau, flavonoids.

\section{Introduction}

Durum wheat semolina quality is an essential factor in the development of pasta, which is a "made in Italy" typical product both in national and international market [1]. Many Italian companies, especially in the Apulia region, try to optimize grinding technologies in order to preserve quality and develop the product functionality. In fact durum wheat is the raw material, whose convertion into semolina, leads to the main ingredient in the production of bread and fresh and dry pasta. The final product is the result of the synergy of two main factors: the choice of the best raw material and the high technology of the productive process. The great availability of raw materials, which are selected from qualitative most interesting origins, allows the production of "customized" semolina perfectly responsive to customer demand and expectance. The grinding process, which has not changed along 100 years, has been improved with the introduction of forefront technological process such as

Corresponding author: Pamela Vignolini, Ph.D, research fields: food chemistry, analytical chemistry, commodity science. decortication and optic selection, which contributed to the improvement of the whole productive process, by increasing both product quality and its healthiness. The semolina quality enhancement, which is related to bread and pasta quality, needs a careful study of secondary metabolites with high biologic and functional properties, such as carotenoids and polyphenol compounds [2-4].

The present study involved the analysis of semolina several samples, obtained through three different grinding processes: conventional grinding, conventional stone grinding and stone grinding with innovative process, in different stages of picking. Carotenoids and polyphenolic compounds content was monitored in correlation with the grinding technologies and the role of these compounds was related to the antioxidant properties of the product.

\section{Materials and Methods}

\subsection{Samples}

Two batches of durum wheat semolina were obtained through three types of grinding: conventional grinding, conventional stone grinding and stone 
grinding with an innovative process optimized by the Casillo Group, in different stages of picking (initial, intermediate, final and coacervation) (Table 1).

\subsection{Extraction}

Carotenoids: $10 \mathrm{~g}$ semolina was extracted with 100 $\mathrm{mL}$ acetone, cold sonicated for $30 \mathrm{~min}$. The sample was centrifuged for $5 \mathrm{~min}$ at $5,000 \mathrm{rpm}$, the supernatant has been dry evaporated with a Rotovapor and the residue was dissolved in $5 \mathrm{~mL}$ acetone. The extracts were used for HPLC-DAD analysis.

Polyphenols: $10 \mathrm{~g}$ semolina was extracted with 50 $\mathrm{mL}$ of 70:30 EtOH/ $\mathrm{H}_{2} \mathrm{O}$ at $\mathrm{pH} 3.2$ (by $\mathrm{HCOOH}$ ). The samples were shaken for $24 \mathrm{~h}$, centrifuged for $5 \mathrm{~min}$ at $1,400 \mathrm{rpm}$ and used for HPLC-DAD (high performance liquid chromatography/diode array detection) analysis.

\subsection{Standards}

Authentic standards of indoleacetic acid, chlorogenic acid and Kaempferol 3-glucoside, and Folin-Ciocalteau reagent, were purchased from Sigma-Aldrich (St. Louis, USA). $\beta$-carotene standard was purchased from Extrasynthese (Lione, Francia).

\subsection{Solvents}

All solvents used were of HPLC grade purity (BDH Laboratory Supplies, Poole, United Kingdom).

\subsection{HPLC/DAD Analysis}

Qualy-quantitative analyses of carotenoids and polyphenols were carried out using an HP 1100 liquid chromatography equipped with a DAD detector and managed by an HP 9000 workstation (Agilent Technologies, Palo Alto, CA, USA). Compounds were separated using a $250 \times 4.6 \mathrm{~mm}$ i.d., $5 \mu \mathrm{m}$ LUNA $\mathrm{C}_{18}$ column (Phenomenex, USA). UV/Vis spectra were recorded in the 190-600 $\mathrm{nm}$ range and the chromatograms were acquired at 250, 280, 330, 350 and $450 \mathrm{~nm}$. The samples were analyzed by gradient elution at a flow rate of $0.8 \mathrm{~mL} / \mathrm{min}$. The mobile phase for carotenoids was a multistep linear solvent gradient system (solvent A: acetone, solvent $\mathrm{B}: \mathrm{H}_{2} \mathrm{O}, \mathrm{pH} 3.2$ by $\mathrm{HCOOH}$ ), starting from $80 \%$ acetone up to $100 \%$ in $30 \mathrm{~min}$. Polyphenols were eluted using the following gradient: from $90 \% \mathrm{H}_{2} \mathrm{O}$ (adjusted to $\mathrm{pH} 3.2$ by $\mathrm{HCOOH}$ ) to $100 \% \mathrm{CH}_{3} \mathrm{CN}$ in $40 \mathrm{~min}$.

\subsection{Quantitative Analysis}

Quantification of individual polyphenolic compounds was directly performed by HPLC-DAD using a five-point regression curve $\left(r^{2} \geq 0.998\right)$ in the range of $0-30 \mu \mathrm{g}$ on the basis of authentic standards. In particular, flavonols were determined at $350 \mathrm{~nm}$ using kaempferol 3-O-glucoside as reference compound while caffeic acid derivatives were determined at $330 \mathrm{~nm}$ using clorogenic acid as reference compound and indoleacitic acid derivative at $280 \mathrm{~nm}$ using indoleacetic acid. Carptenoids were determined at $450 \mathrm{~nm}$ using $\beta$-carotene as reference compound. In all cases, actual concentrations of the derivatives were calculated after applying corrections for differences in molecular weight.

\subsection{Total Phenolic Content}

The total phenolic content was determined using the Folin-Ciocalteu method, described by Singleton et al. [5] and slightly modified according to Dewanto et al. [6]. To $125 \mu \mathrm{L}$ of the suitably diluted sample extract, $0.5 \mathrm{~mL}$ of deionized water and $125 \mu \mathrm{L}$ of the Folin-Ciocalteu reagent was added. The mixture was kept for $6 \mathrm{~min}$ and then $1.25 \mathrm{~mL}$ of $7 \%$ aqueous $\mathrm{Na}_{2} \mathrm{CO}_{3}$ solution was added. The final volume was adjusted to $3 \mathrm{~mL}$ with water. After $90 \mathrm{~min}$, the absorption was measured at $760 \mathrm{~nm}$ against water as a blank. The amount of total phenolics is expressed as gallic acid equivalents (GAE, mg gallic acid/100 g sample) through the calibration curve of gallic acid. The calibration curve ranged from 20 to $500 \mu \mathrm{g} / \mathrm{mL}$ $\left(\mathrm{R}^{2}=0.9969\right)$.

\section{Results and Discussion}


Both elution and characterization were optimized for these matrices. By HPLC-DAD chromatograms at different wavelengths were obtained and therefore the metabolites could be identified. As an example, Fig. 1 reports the chromatographic profile of the acetone extract of a semolina sample at $450 \mathrm{~nm}$, which is the maximum absorption wavelength for carotenoids.

In Fig. 2, the UV-Vis spectra of some carotenoids are reported.

As reported in previous studies [2,7], lutein was the main carotenoid identified in all samples, as shown in Tables 2 and 3. Its content changed between 2.48 and
$6.73 \mathrm{mg} / \mathrm{g}$. In particular, lutein content is always greater in the starting grinding phase, regardless of the type of grinding used, while almost always stone grinding highlighted the highest carotenoids content.

In the semolina samples, other than carotenoids, even flavonoids and an indoleacetic acid derivatives were pointed out (Tables 4 and 5); the indoleacetic acid derivative is the main compound (0.78-0.108 $\mathrm{mg} / \mathrm{g}$ ). The highest polyphenol content was found in stone grinded and with stone cylinder with respect to the conventional grinding process.

Table 1 Samples analyzed.

\begin{tabular}{lll}
\hline & Types of grinding & Stages of picking \\
\hline $\begin{array}{l}\text { Durum wheat semolina } \\
1^{\circ}-2^{\circ} \text { batche }\end{array}$ & Stone grinded & Coacervation \\
& & Initial \\
& & Intermediate \\
& & Final \\
$\begin{array}{l}\text { Durum wheat semolina } \\
1^{\circ}-2^{\circ} \text { batche }\end{array}$ & Conventional with stone cylinder & Initial \\
& & Intermediate \\
& & Final \\
Durum wheat semolina & & Coacervation \\
$1^{\circ}-2^{\circ}$ batche & Conventional & Initial \\
& & Intermediate \\
\hline
\end{tabular}

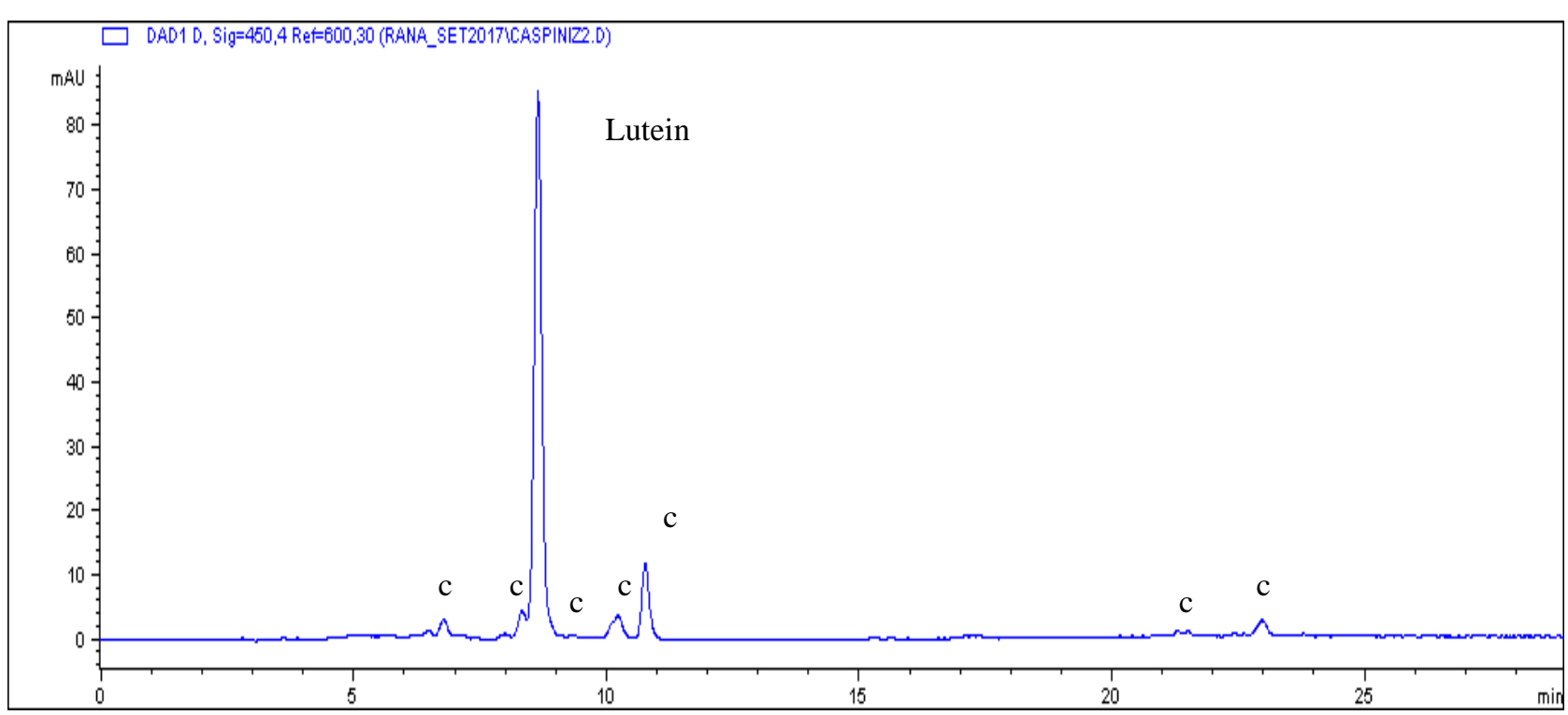

Fig. 1 Chromatographic profile at $450 \mathrm{~nm}$ of an acetone semolina extract $(\mathrm{C}=$ carotenoid). 


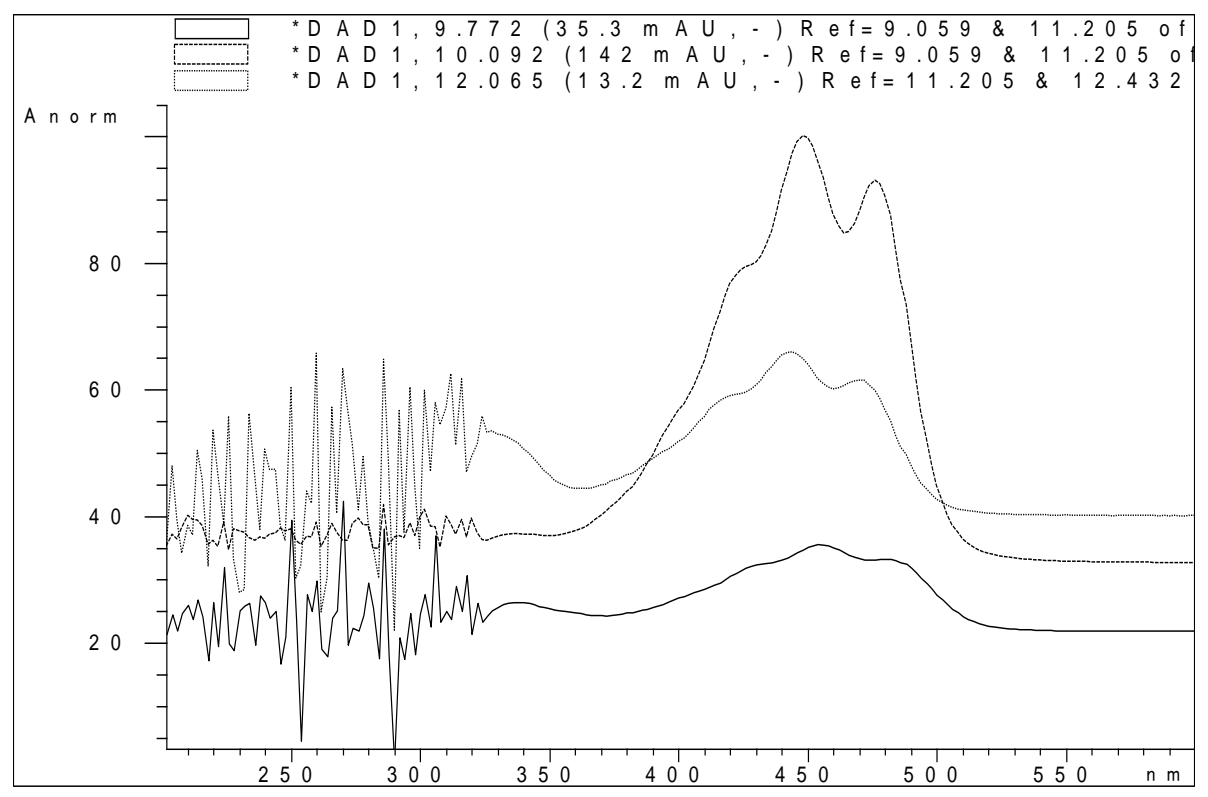

Fig. 2 UV-Vis spectra of some carotenoids in semolina acetone extracts.

Table 2 Carotenoids and lutein content in semolina samples, obtained through three different grinding processes: conventional grinding, conventional stone grinding and stone grinding with innovative process, in different stages of picking ( $1^{\circ}$ batche June 2017). Data are the mean of three determinations (standard deviation < $3 \%$ ).

\begin{tabular}{llll}
\hline & $\begin{array}{l}\text { Total carotenoids } \\
(\mathrm{mg} / \mathrm{kg})\end{array}$ & $\begin{array}{l}\text { Lutein } \\
(\mathrm{mg} / \mathrm{kg})\end{array}$ & $\begin{array}{l}\text { Carotenoids } \\
(\mathrm{mg} / 100 \mathrm{~g})\end{array}$ \\
\hline Stone grinded semolina & & & 0.70 \\
\hline Coacervation & 6.96 & 5.45 & 0.54 \\
Final & 5.38 & 4.16 & 0.59 \\
Intermediate & 5.94 & 4.59 & 0.81 \\
Initial & 8.05 & 6.37 & 0.47 \\
\hline Conventional grinded with stone cylinder semolina & & & 0.50 \\
\hline Coacervation & 4.72 & 3.60 & 0.52 \\
Final & 5.02 & 3.86 & 0.51 \\
Intermediate & 5.21 & 3.93 & 0.53 \\
Initial & 5.12 & 3.93 & 0.56 \\
Conventional grinded semolina & & & 0.72 \\
\hline Coacervation & 5.31 & 3.96 & 0.86 \\
Final & 5.64 & 4.22 & 5.48 \\
Intermediate & 7.19 & 6.73 & \\
Initial & 8.65 & & \\
\hline
\end{tabular}

Table 3 Carotenoids and lutein content in semolina samples, obtained through three different grinding processes: conventional grinding, conventional stone grinding and stone grinding with innovative process, in different stages of picking ( $2^{\circ}$ batche September 2017). Data are the mean of three determinations (standard deviation $<3 \%$ ).

\begin{tabular}{llll}
\hline & $\begin{array}{l}\text { Total carotenoids } \\
(\mathrm{mg} / \mathrm{kg})\end{array}$ & $\begin{array}{l}\text { Lutein } \\
(\mathrm{mg} / \mathrm{kg})\end{array}$ & $\begin{array}{l}\text { Carotenoids } \\
(\mathrm{mg} / 100 \mathrm{~g})\end{array}$ \\
\hline Stone grinded semolina & & & 0.52 \\
\hline Coacervation & 5.18 & 3.93 & 0.5 \\
Final & 5.05 & 3.76 & 0.51 \\
Intermediate & 5.12 & 3.93 & 0.54 \\
Initial & 5.45 & 4.06 & \\
\hline
\end{tabular}


Table 3 to be continued

\begin{tabular}{llll}
\hline Conventional grinded with stone cylinder semolina & & & \\
\hline Coacervation & 3.66 & 2.67 & 0.37 \\
Final & 3.60 & 2.67 & 0.36 \\
Intermediate & 3.37 & 2.48 & 0.34 \\
Initial & 3.76 & 2.74 & 0.38 \\
\hline Conventional grinded semolina & & & 0.47 \\
Coacervation & 4.69 & 3.33 & 0.45 \\
Final & 4.52 & 3.20 & 0.49 \\
Intermediate & 4.88 & 3.73 & 0.51 \\
Initial & 5.08 & 3.96 & \\
\hline
\end{tabular}

Table 4 Flavonoids and IAA derivative content in semolina samples, obtained through three different grinding processes: conventional grinding, conventional stone grinding and stone grinding with innovative process, in different stages of picking ( $1^{\circ}$ batche June 2017), IAA = indoleacetic acid. Data are the mean of three determinations (standard deviation $\left.<3 \%\right)$.

\begin{tabular}{lll}
\hline & IAA derivative $(\mathrm{mg} / \mathrm{g})$ & Total flavonoids $(\mathrm{mg} / \mathrm{g})$ \\
\hline Stone grinded semolina & & \\
\hline Coacervation & 0.098 & 0.024 \\
Final & 0.103 & 0.025 \\
Intermediate & 0.103 & 0.026 \\
Initial & 0.106 & 0.025 \\
\hline Conventional grinded with stone cylinder semolina & & \\
\hline Coacervation & 0.094 & 0.011 \\
Final & 0.078 & 0.009 \\
Intermediate & 0.098 & 0.010 \\
Initial & 0.100 & 0.012 \\
\hline Conventional grinded semolina & & \\
\hline Coacervation & 0.101 & 0.014 \\
Final & 0.106 & 0.014 \\
Intermediate & 0.105 & 0.014 \\
Initial & 0.106 & 0.014 \\
\hline
\end{tabular}

Table 5 Flavonoids and IAA derivative content in semolina samples, obtained through three different grinding processes: conventional grinding, conventional stone grinding and stone grinding with innovative process, in different stages of picking $\left(2^{\circ}\right.$ batche September 2017), IAA = indoleacetic acid. Data are the mean of three determinations (standard deviation $\left.<3 \%\right)$.

\begin{tabular}{lll}
\hline & IAA derivative $(\mathrm{mg} / \mathrm{g})$ & Total flavonoids $(\mathrm{mg} / \mathrm{g})$ \\
\hline Stone grinded semolina & & \\
\hline Coacervation & 0.105 & 0.024 \\
Final & 0.108 & 0.023 \\
Intermediate & 0.107 & 0.022 \\
Initial & 0.106 & 0.022 \\
\hline Conventional grinded with stone cylinder semolina & & \\
\hline Coacervation & 0.097 & 0.007 \\
Final & 0.100 & 0.007 \\
Intermediate & 0.100 & 0.006 \\
Initial & 0.098 & 0.006 \\
\hline Conventional grinded semolina & & \\
\hline Coacervation & 0.100 & 0.014 \\
Final & 0.105 & 0.015 \\
Intermediate & 0.103 & 0.015 \\
Initial & 0.103 & 0.014 \\
\hline
\end{tabular}



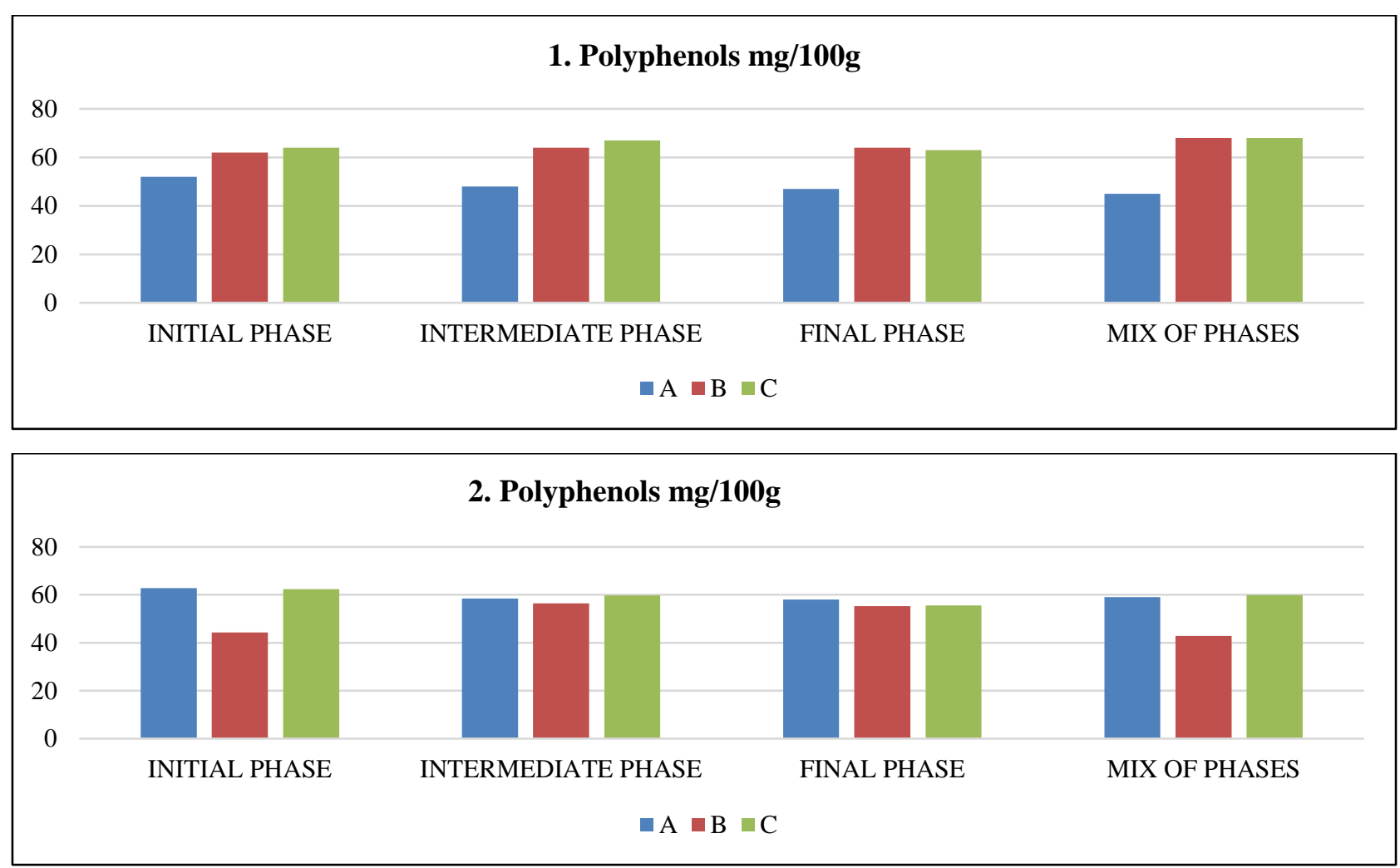

Fig. 3 Total phenolic content (mg/100 g, Folin-Ciocalteau method).

$1=1^{\circ}$ batche june 2017; $2=2^{\circ}$ batche September 2017; $\mathrm{A}=$ conventional grinded semolina; $\mathrm{B}=$ conventional grinded with stone cylinder semolina; $\mathrm{C}=$ stone grinded semolina.

Data are the mean of three determinations (standard deviation $<3 \%$ ).

The presence of antioxidant secondary metabolites has been evaluated with in vitro spectrophotometric techniques such as the Folin Ciocalteu method (Fig. 3). With this test the total antioxidant capacity of biocomponents can be deduced.

Fig. 3 shows that the sample obtained with stone grinding almost always has slightly higher values than the one obtained with conventional grinding with stone cylinder and both technologies are almost always better than conventional technology in all sampling phases.

The data show that the technology influences the quality of the product also for the content in secondary bioactive metabolites, particular role is correlated with the quality of the raw material.

Further tests are being carried out to optimize all the milling phases using semolina of different quality characteristics. The work will continue using the flour for making pasta and evaluating the functional properties of the obtained pasta.

\section{Acknowledgements}

We thank Casillo Group for providing us the samples, in the context of the research "Verifica delle caratteristiche chimiche, della stabilità qualitativa e delle proprietà antiossidanti di campioni di semola e di pasta derivata, provenienti dal processo di macinazione con rulli rivestiti in pietra della Molino Casillo S.p.A”.

\section{References}

[1] Kissing K. L., Dyck E., Russell J., Clark L., and Dawson J. C. 2017. "Evaluation of Wheat and Emmer Varieties for Artisanal Baking, Pasta Making, and Sensory Quality.” Journal of Cereal Science, 74: 19-27.

[2] Mellado-Ortega, E., and Hornero-Méndez, D. 2016."Carotenoid Evolution during Short-Storage Period of Durum Wheat (Triticum turgidum conv. durum) and Tritordeum (Tritordeum Ascherson et Graebner) Whole-Grain Flours." Food Chemistry 192: 714-23. 
[3] Pinzino, C., Nanni, B., and Zandomeneghi, M. 1999. "Aging, Free Radicals, and Antioxidants in Wheat Seeds". J. Agric. Food Chem. 47:1333-9.

[4] Rao, A. V., and Rao, L. G. 2007. "Carotenoids and Human Health". Pharmacol. Res. 55: 207-16.

[5] Singleton, V. L., Orthofer, R., and Lamuela-Raventos, R. M. 1999. "Analysis of Total Phenols and Other Oxidation Substrates and Antioxidants by Means of the Folin-Ciocalteu Reagent.” Meth. Enzymol. 299: 152-78.

[6] Dewanto, V., Wu, X., Adom, K. K., and Liu, R. H. 2002.
"Thermal Processing Enhances the Nutritional Value of Tomatoes by Increasing Total Antioxidant Activity." $J$. Agric. Food Chem. 50: 3010-4.

[7] Mellado-Ortega E., Atienza S.G., Hornero-Méndez D. 2015. "Carotenoid Evolution during Postharvest Storage of Durum Wheat (Triticum turgidum conv. durum) and Tritordeum (Tritordeum Ascherson et Graebner) Grains". Journal of Cereal Science 62: 134-42. 